\title{
Experimental investigation of the repelling force from RF carpets
}

\author{
A. Hamaker ${ }^{1,2}$, M. Brodeur ${ }^{1 *}$, J.M. Kelly ${ }^{1}$, J. Long ${ }^{1}$, \\ C. Nicoloff ${ }^{1,3}$, S. Ryan ${ }^{1}$, B.E. Schultz ${ }^{1}$, P. Schury ${ }^{4}$, and M. Wada ${ }^{4}$ \\ ${ }^{1}$ University of Notre Dame, Department of Physics, Notre Dame IN, 46556, USA \\ ${ }^{2}$ Hanover College, Department of Physics, Hanover IN, 47243, USA \\ ${ }^{3}$ Department of Physics, Wellesley College, Wellesley, MA 02481 USA and \\ ${ }^{4}$ Wake Nuclear Science Center (WNSC) / RNB(Radioactive Nuclear Beam) group, \\ Institute of Particle and Nuclear Studies, \\ High Energy Accelerator Research Organization, Wako, Saitama, 351-0198, Japan
}

(Dated: April 18, 2016)

\begin{abstract}
The theoretical description for the ion motion above a radio frequency carpet was proposed in [S. Schwarz, Int. J of Mass Spec. 299, 71 (2011)], and using this description, the maximal repelling force created by the RF carpet that leads to stable ion motion can be determined. The predicted changes in the repelling force were experimentally tested for different RF amplitudes, helium gas pressures, and electric push field strength that the RF carpet is counteracting. We observed good overall agreement between the experimental and the theoretical description.
\end{abstract}

PACS numbers: 29.27.Ac,29.27.Eg,34.50.Bw

* corresponding author: mbrodeur@nd.edu 


\section{INTRODUCTION}

With the continued emergence of new radioactive ion beam facilities worldwide [1], there is a high potential for answering pressing questions ranging from the creation of heavy nuclei to the structure of exotic nuclei. Among the two major types of production methods [1], Isotope Separator On-Line (ISOL) and in-flight, the chemistry-independent nature of the latter method allows for the delivery of a broader range of elements. However, the high energy and momentum spread of the produced beams from the in-flight method seem to be at odds with the requirements for low energy experiments such as high precision mass measurements and laser spectroscopy [2]. Fortunately, the development of gas cells [3] has bridged the gap. While these gas cells vary in design, a common feature of many of them is the use of so-called radio-frequency $(\mathrm{RF})$ carpets [4]. The RF carpet can take various forms, from copper rings with a rigid or semi-rigid backing [5-7] to individual electrodes spaced by ceramic insulators $[8,9]$. However, these various configurations all serve the dual purpose of repelling the ions from the gas cell walls while transporting them towards an extraction orifice. Other than nuclear physics, RF carpets can be used in other areas of research including analytical chemistry. For instance, it was recently shown that a combination of an RF carpet and a DC carpet can form a simple electrospray interface $[10,11]$.

There are currently two methods of using RF carpets for transporting ions in gas volumes: the traditional method [4], for which a static electric potential of decreasing strength towards the extraction orifice is applied on the individual electrodes, and the ion surfing method [12], which replaces this static potential with a traveling wave. The common feature of both methods is the application of RF signals on the carpet electrodes to provide a repelling force. The ion motion above the carpet and the stability conditions for that motion were investigated, leading to a theoretical description of the maximal force on an ion that an RF carpet can balance [13]. The force being balanced can derive from such necessary sources as the drag field that moves the ion along the carpet, or from detrimental sources such as the induced ionization of the buffer gas.

In both situations, knowing how to maximize the repelling force is desirable for both the design and optimal operation of RF carpets. Hence, considering the growing use of RF carpet technology, we have investigated experimentally the behavior of the repelling force as a function of various experimental parameters. In this paper, we report on the results for 


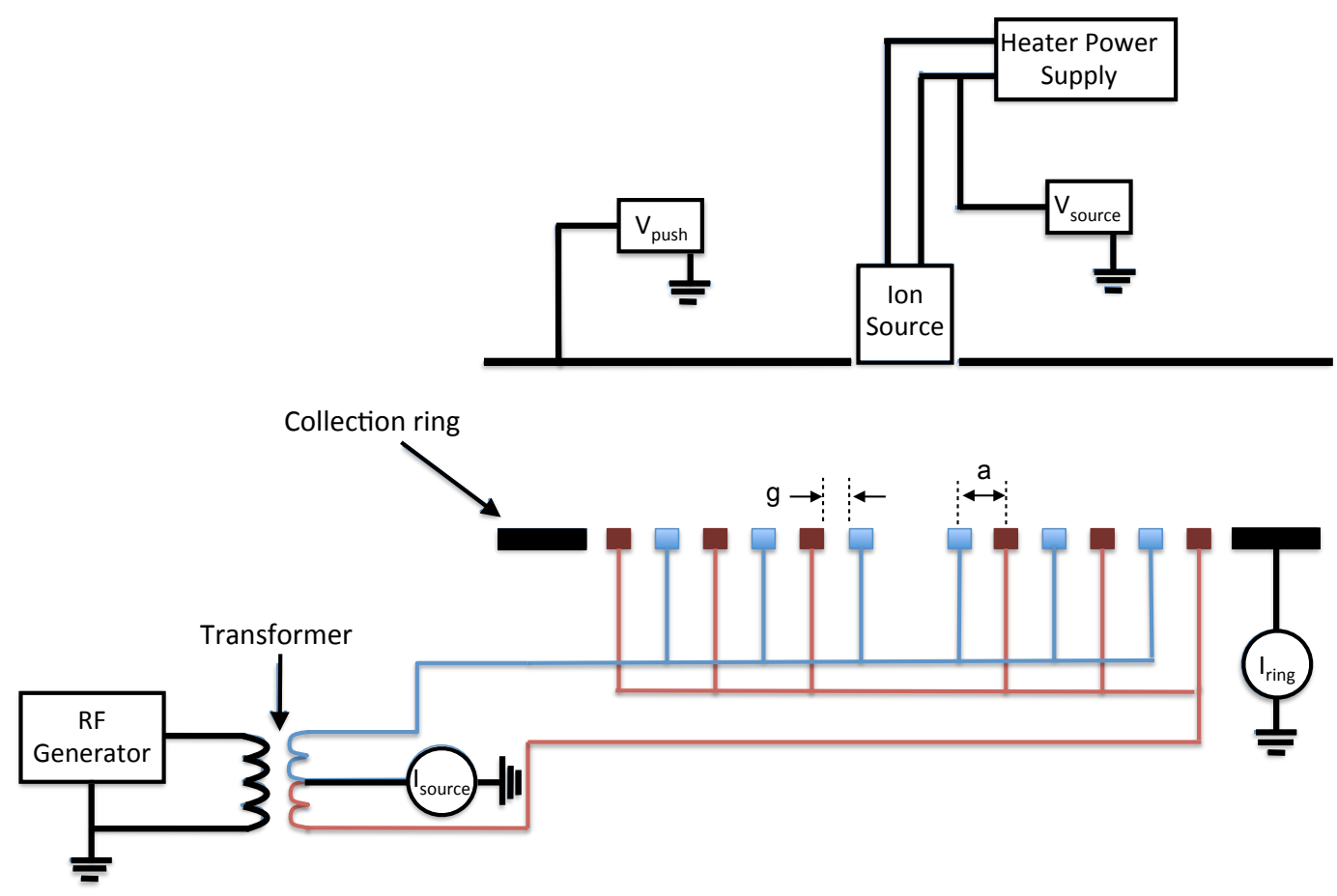

FIG. 1: (Color on-line) Schematic view of experimental set-up. The dark red and light blue squares denote the alternating electrodes of opposite RF phase.

various values of the RF amplitude and the pressure inside the gas cell.

\section{THEORETICAL DESCRIPTION}

An RF carpet is made of a series of closely spaced electrodes on which an alternating potential is applied with a $180^{\circ}$ phase shift between adjacent electrodes (see Fig. 1). The resulting time-dependent electric field creates a repelling force above the electrodes. To keep the ions hovering just above the RF carpet, a "push" force is used to balance the repelling force from the RF. The push force can be created by applying a voltage to a metallic plate above the RF carpet surface.

Using the analytical expression for the electric field produced by an RF carpet [13], the equation of motion of an ion above the carpet can be written as [13]:

$$
\frac{d^{2} y_{\pi}}{d \xi^{2}}=E_{d} e^{-y_{\pi}} \cos (\xi)-E_{p r}-\kappa \frac{d y_{\pi}}{d \xi}
$$

where $\xi=2 \pi f, y_{\pi}=y \pi / a, f$ is the RF frequency, $a$ is the center-to-center distance between 
electrodes (also known as the carpet pitch), and $y$ is the vertical distance from the carpet electrode surface. The dimensionless parameters $E_{d}$ (RF amplitude-dependent), $E_{p r}$ (push field-dependent) and $\kappa$ (pressure-dependent) are defined as:

$$
\begin{array}{r}
E_{d}=\frac{q}{m} \frac{2 V_{R F}}{\gamma \pi^{2} a^{2} f^{2}} \sin (\pi \gamma / 2) \\
E_{p r}=\frac{q}{m} \frac{1}{4 \pi a f^{2}} E_{p} \\
\kappa=\frac{q}{m} \frac{1}{2 \pi f K_{0}} \frac{p / p_{0}}{T / T_{0}}
\end{array}
$$

where $\gamma$ is the ratio of the gap $g$ between adjacent electrodes to their pitch, $V_{R F}$ is the zero-to-peak RF amplitude, $E_{p}$ is the push field, $m$ is the ion mass, $p$ is the gas pressure, $T$ is the gas temperature, with $p_{0}=1013.25 \mathrm{mbar}$ and $T_{0}=273.15 \mathrm{~K}, q$ is the charge of the ion, and $K_{0}$ is the reduced ion mobility in the buffer gas.

As described in [13], Eq. 1 can be solved numerically to obtain the time-dependence of the distance of the ion above the RF carpet. The carpet's maximum sustainable push field as a function of either RF amplitude or pressure can be found from the value of $E_{p r}$ in the case where the ion would just touch the carpet $\left(y_{\pi} \rightarrow 0\right)$.

\section{EXPERIMENTAL SET-UP}

The experimental setup used to perform the measurements includes an RF carpet, a stainless steel plate to provide the push field, and an ion source (see Fig. 2), which are all placed inside the vacuum chamber shown in Fig. 3. The circuitry used to produce the RF and the push field, the ion source power supply, the RF supplies, and instrumentation were placed outside the vacuum chamber.

The vacuum chamber was evacuated with an Edwards nXDS10i $11.4 \mathrm{~m}^{3} / \mathrm{h}$ scroll pump and an Edwards STP301 $300 \mathrm{~L} / \mathrm{s}$ turbo molecular pump in order to minimize residual air contamination in the chamber. The system was pumped down to a base pressure in the $10^{-9}$ mbar range between measurements. During the measurements, Grade 5 ultra-pure helium was injected in the chamber. The gas pressure was monitored using three different gauges: a convection gauge when pumping down the system, a hot cathode gauge at ultra high vacuum conditions, and a baratron gauge for a precise, absolute pressure reading during the measurements. A thermocouple was also used to measure the temperature inside the 


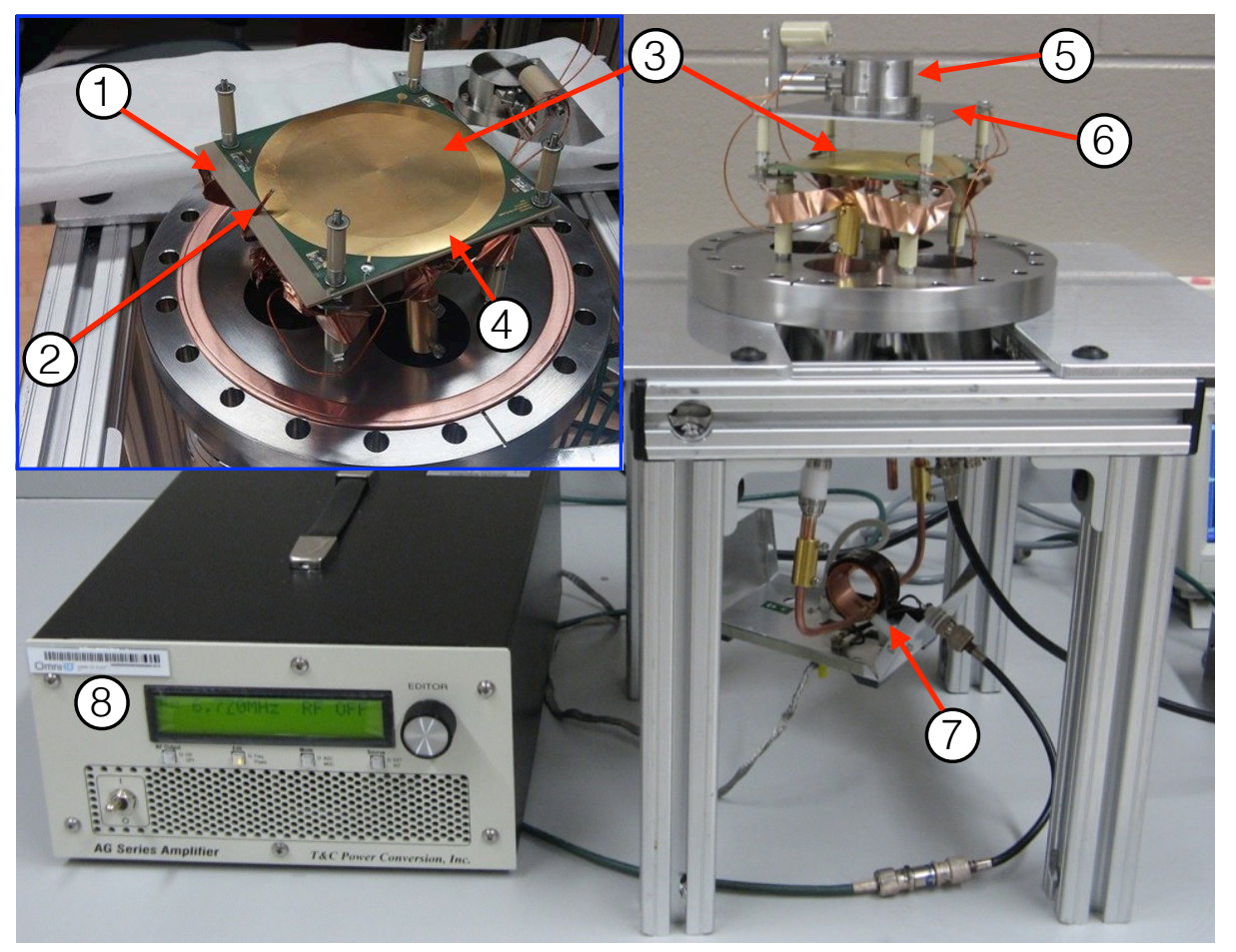

FIG. 2: Photograph of the RF carpet and the circuitry on a holding frame for tuning. (1) sheet of PEEK preventing the carpet from flexing, (2) thermocouple, (3) the RF carpet, (4) the outer ring collecting the transported ions, (5) the ion source, (6) the stainless steel push plate, (7) the $\mathrm{RF}$ circuit with the air core impedance matching transformer, and (8) the RF generator.

chamber (see Fig. 2). In order to avoid RF discharges in the chamber, the RF amplitude was kept below $70 \mathrm{~V}$.

The circuitry used to operate the RF carpet was constructed to maximize the RF frequency on the carpet and maintain a stable RF amplitude. In order to maximize the transfer of power from the generator to the carpet, the RF signal from a AC1020 175W low frequency amplifier from T\&C Power Conversion was impedance matched using an air core impedance matching transformer (see Fig. 2). The transformer was made of a single turn of a $2 \mathrm{~cm}$ large and $4 \mathrm{~cm}$ diameter copper tube while the primary was made of 9 turns of 14 gauge copper wire. To minimize resistive losses, only copper was used to carry the RF in the circuitry. The power provided by the RF generator did not exceeded $5 \mathrm{~W}$.

The push field $E_{p}$ used to bring the ions close to the carpet surface was produced by applying a positive potential to a stainless steel plate. A commercial $\mathrm{K}^{+}$surface ion source 


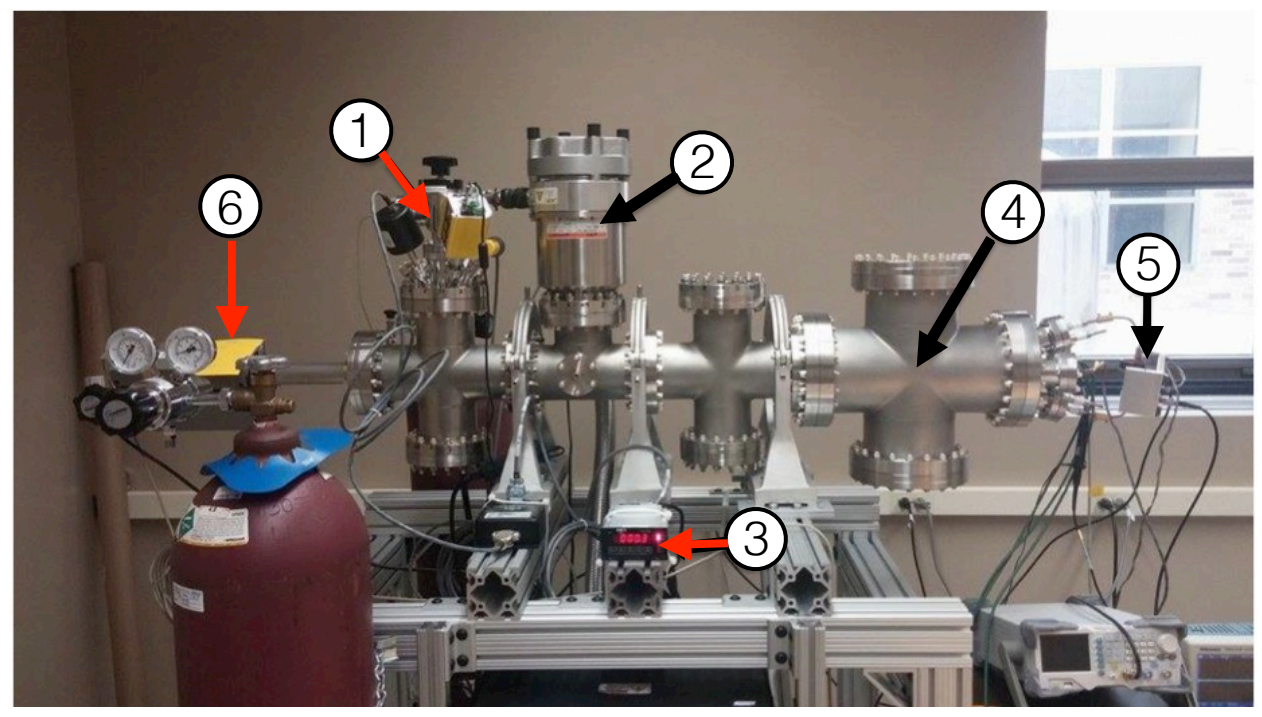

FIG. 3: Photograph of the experimental setup. (1) is the hot cathode gauge, (2) the turbo molecular pump, (3) the baratron gauge controller, (4) the vacuum chamber where the RF is set up, (5) the $\mathrm{RF}$ circuit, and (6) the residual gas analyzer.

from HeatWave Labs [14] was used, which contains a molybdenum filament embedded in alumina surrounded by a molybdenum body. The emission surface consists of a porous tungsten disk over which an alumino-silicate compound of the alkali of interest is fused. The purity of the ion beam produced by the source was confirmed by directing the beam towards a SRS RGA200 residual gas analyzer with its filament turned off. Only ${ }^{39} \mathrm{~K}^{+}$and ${ }^{41} \mathrm{~K}^{+}$at their respective natural abundance of $93 \%$ and $7 \%$ were observed.

A $1.6 \mathrm{~mm}$ thick sheet of PEEK was placed under the RF carpet in order to prevent the carpet from flexing. The RF carpet was manufactured by Compunetics [15] with a pitch of $0.16 \mathrm{~mm}$ and a gap between adjacent electrodes of $0.08 \mathrm{~mm}$, resulting in a gap-to-pitch ratio $\gamma=0.5$. The distance between the plate and the RF carpet was $2.8 \mathrm{~cm}$.

For typical operation at a helium pressure of $\sim 100$ mbar, a heating current $I_{\text {heat }} \approx 3.00 \mathrm{~A}$ and heating voltage $V_{\text {heat }} \approx 7.6 \mathrm{~V}$ was needed to obtain $I_{\text {source }} \approx 100 \mathrm{pA}$. It should be noted that a degradation of the ion source performance was apparent when $I_{\text {heat }}$ was maintained above $3.2 \mathrm{~A}$ over long periods of time. The heater current for the ion source was provided by a $60 \mathrm{~V}, 9$ A DC power supply. The resulting ion current hitting the carpet could be measured through the center tap of the secondary part of the transformer while the RF was turned off (see Fig. 2). The ions produced were focused and directed toward the RF carpet 
using the stainless steel plate biased to $V_{p}<V_{\text {source. }}$. The RF carpet was set up in such a way as to carry the ions by outward diffusion to a collection ring located on the edge of the carpet where the current $I_{\text {ring }}$ could be measured (see Fig. 1 and 2). Both currents $\left(I_{\text {source }}\right.$ and $I_{\text {ring }}$ ) were measured using a Keithley 6514 electrometer. A 25 mm radius collection copper plate was placed $12 \mathrm{~mm}$ under the carpet central hole to record ions leaking through the hole. Throughout the measurements, no significant leaking current was observed (less than $5 \mathrm{pA})$.

\section{MEASUREMENT METHOD}

The maximum push field that an RF carpet can sustain before the ion hits the carpet can be tested by measuring the fraction of the ion beam that diffused across the carpet under various conditions when only the RF is applied on the carpet electrodes. For the measurements, the push field was simply calculated as: $E_{p}=V_{p} / d$, where $d=2.8 \mathrm{~cm}$ is the distance between the stainless steel plate (providing the push field) and the RF carpet. The gas pressure, push field strength, and the RF amplitude were all varied one at a time. In order to make consistent measurements, a fixed value for the source current, $I_{\text {source }}$, and the temperature $T\left(\sim 100 \mathrm{pA}\right.$ and $\left.60^{\circ} \mathrm{C}\right)$ were maintained throughout data collection. This was achieved by adjusting $V_{\text {source }}$ and the heater current, $I_{\text {heat }}$, used to create the ions. A current of $100 \mathrm{pA}$ was chosen to minimize possible space charge effects while having sufficient current for a precise measurement, and $60{ }^{\circ} \mathrm{C}$ was the measured temperature where a $100 \mathrm{pA}$ current was readily produced. It should be noted that the original $23{ }^{\circ} \mathrm{C}$ room temperature was raised to $60{ }^{\circ} \mathrm{C}$ due to the heating of the ion source. The application of the RF signal does not change the chamber temperature by more than 1 to 2 degrees. It should be noted that the thermal conductivity of gas, which will set the temperature gradient from the ion source to the chamber wall, is independent of the gas pressure as long as the mean free path is much smaller than the dimension of the chamber [16]. At our operating pressure range ( $p$ $>1 \mathrm{mbar}$, the mean free path is smaller than $0.1 \mathrm{~mm}$, which is much smaller than the size of the vacuum chamber. Hence one can assume that the thermal conductivity of the helium gas was pressure-independent and a constant temperature gradient from the ion source to the thermocouple and across the carpet was maintained.

Measurements were performed once the source current and temperature had stabilized, 

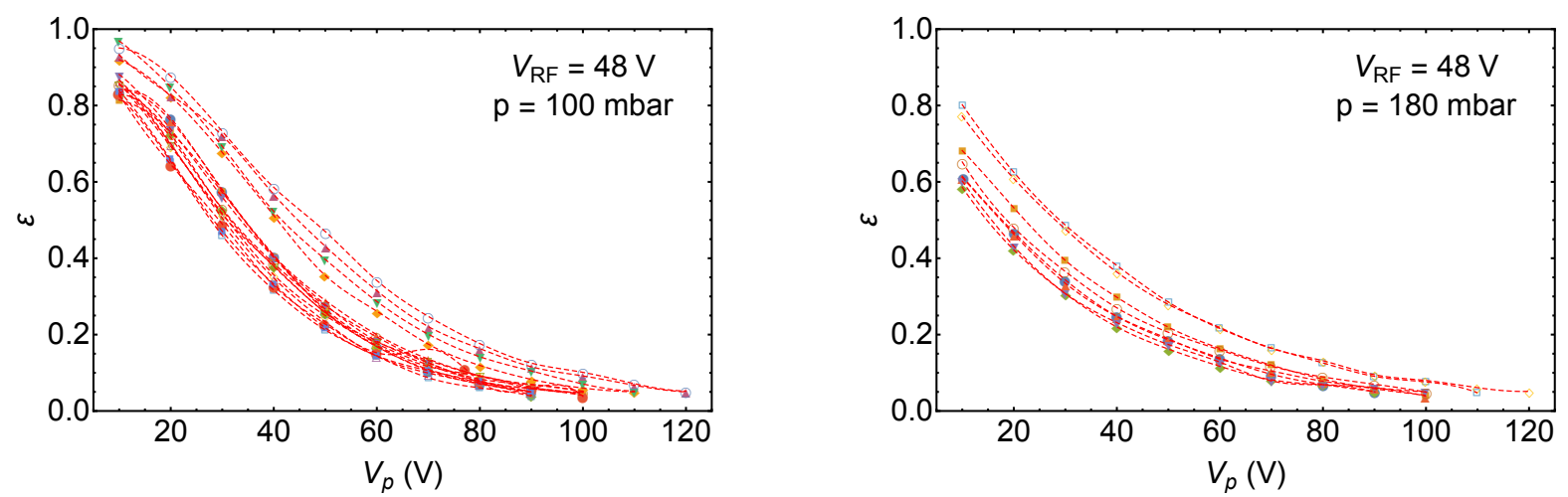

FIG. 4: Variation of the survival fraction with the potential on the metallic plate facing the RF carpet for a $48 \mathrm{~V} \mathrm{RF}$ amplitude and at 100 mbar (left) and 180 mbar (right) helium gas pressure. The different symbols indicate measurements done on different days. The dashed red lines represent the spline interpolation used to determine $E_{p, 50 \%}$.

after a $\sim 45$ minute long warm up period. Starting with $V_{p}$ at approximately $10 \mathrm{~V}$, a measurement of $I_{\text {source }}$ was recorded. The RF was then turned on and $I_{\text {ring }}$ was measured. The RF was then turned off, and another measurement of $I_{\text {source }}$ was recorded to ensure that the source current did not change while $I_{\text {ring }}$ was being recorded. Next, $V_{p}$ was increased by $10 \mathrm{~V}$ and $V_{\text {source }}$ was increased in order to maintain a value for $I_{\text {source }} \sim 100 \mathrm{pA}$. The process of recording $I_{\text {source }}, I_{\text {ring }}$, and $I_{\text {source }}$ was repeated until $I_{\text {ring }}$ was less than or equal to $5 \mathrm{pA}$. During the measurements, the currents would typically fluctuate by $2 \mathrm{pA}$ or less. Sufficient time before a measurement of $I_{\text {ring }}$ was allotted to let the ion current stabilize. At lower values of $V_{p}$, when most of the ions were reaching the collection ring by diffusion, a wait time of 10 seconds for the current to stabilize was allotted. With increasing voltage, fewer ions will make it to the collection ring, decreasing the required waiting time down to approximately 5 seconds.

The process was repeated for different RF amplitudes or pressures in the chamber. The quantity of interest that was calculated after the measurements was the ion survival fraction which is given by:

$$
\varepsilon=\frac{I_{\text {ring }}}{I_{\text {source }}}
$$

In order to evaluate time-to-time variations in $\varepsilon$, each measurement was repeated multiple times, on separate dates. Fig. 4 shows the behavior of the survival fraction as a function of 
the push voltage at 100 mbar, with $V_{R F}=48 \mathrm{~V}$ for all 16 measurements performed and at 180 mbar, with $V_{R F}=48 \mathrm{~V}$ for all 8 measurements performed. Day-to-day changes in the survival fraction possibly coming from a change in the RF circuit performance were observed and can be seen in Fig. 4. While the survival fraction was observed to steadily decreases with increasing potential, an indication for a leveling off at low potential for optimal pressure and RF amplitude can be observed (see Fig. 4). The decrease in survival fraction is due to the greater number of ions being pushed to the carpet electrodes due to the increasing electric field strength.

\section{RESULTS}

The primary goal of the investigation was to experimentally test the maximum push field behavior predicted by the numerical solution of equation (1) at different values for the gas pressures and RF amplitudes. Since an unambiguous measurement of the maximum push field would be difficult, we instead calculated $E_{p, 50 \%}$, which is the push field when the survival fraction at $V_{p}=10 \mathrm{~V}$ is halved, and then plotted this data to see if it agreed with the solution of eq. (1). To facilitate the determination, the data was interpolated using a spline interpolation as shown in Fig. 4. The measurements were performed at pressures ranging from 20 mbar to 200 mbar at 20 mbar intervals, with push fields ranging from $\sim 1$ $\mathrm{V} / \mathrm{cm}$ to $\sim 20 \mathrm{~V} / \mathrm{cm}, \mathrm{RF}$ frequency of $6.8 \mathrm{MHz}$, and RF amplitudes ranging from $\sim 5 \mathrm{~V}$ to $\sim 70 \mathrm{~V}$

\section{A. Push field vs. RF amplitude}

The RF amplitude measurements were recorded between 6 and 13 times at a pressure of 100 mbar. Fig. 5 shows $E_{p, 50 \%}$ versus the RF amplitude overlaid with the survival fractions at $V=10 \mathrm{~V}$ (called $\varepsilon_{10 \mathrm{~V}}$ later) for these amplitudes. The behavior of $\varepsilon_{10 \mathrm{~V}}$ matches closely the behavior of $E_{p, 50 \%}$ with RF amplitudes; both start low at low RF amplitudes, rise with $V_{R F}$ and plateau beyond $V_{R F}=40 \mathrm{~V}$. Note that a survival fraction of $\varepsilon_{10 \mathrm{~V}}=1$ is never reached. This might be due to the extended diffusion time, which would result in more ions being lost from collisions directing them towards the carpet electrodes. This situation would represent a worse case scenario as in normal RF carpet operation either a potential gradient 

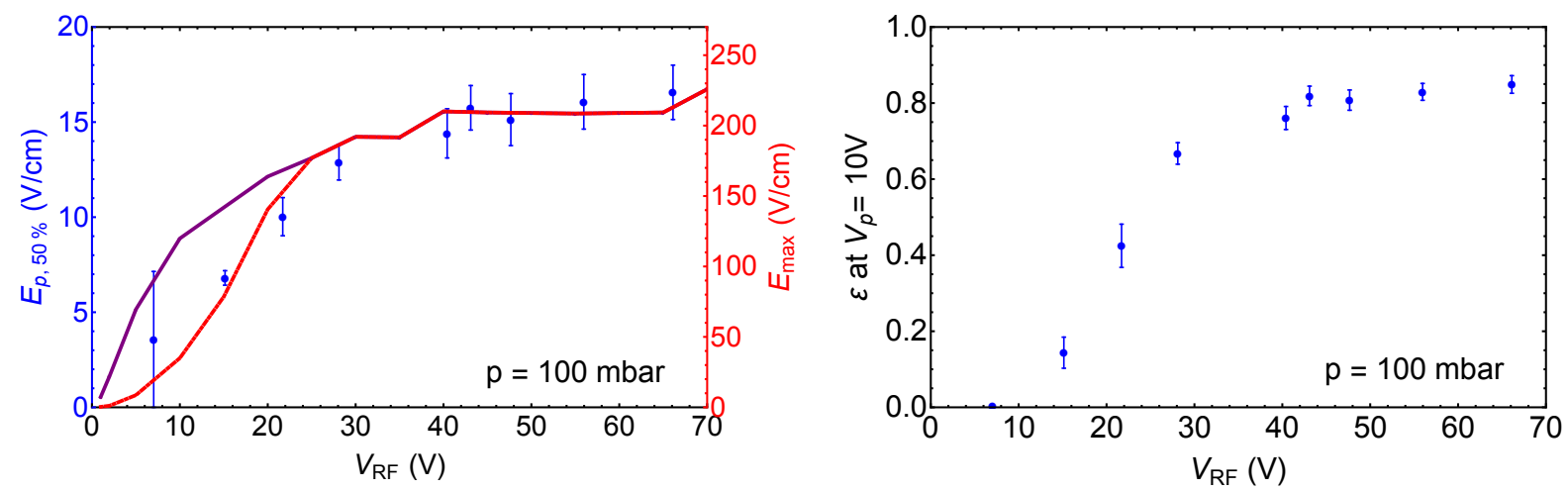

FIG. 5: Left: $E_{p, 50 \%}$ (circles) as a function of RF amplitude. The purple curve is the maximum push field $E_{\max }$, when $y_{\pi}=0$, obtained by solving numerically eq. 1 . The red curve is similar to the solid curve but with the additional requirement that the average distance of the ion above the carpet must be above $0.57 \mathrm{~mm}$. Right: $\varepsilon_{10 \mathrm{~V}}$ as a function of RF amplitude. Both data sets were taken at a pressure of 100 mbar.

or a traveling wave would carry the ions along the carpet, reducing the time spent above the surface of the carpet.

The behavior of the experimental data was investigated by solving Eq. 1 numerically. To solve the equation we assumed the ion started at $y=5 \mathrm{~mm}$ above the carpet and with no initial speed. The other parameters were $m / q=39 \mathrm{amu} / \mathrm{e}, f=6.8 \mathrm{MHz}, a=0.16 \mathrm{~mm}, \gamma$ $=0.5, K_{0}=21.6 \mathrm{~cm}^{2} /(\mathrm{Vs})[17]$, and $T=60^{\circ} \mathrm{C}$. At each RF amplitude Eq. 1 was solved for $y$ numerically with increasing values of $E_{p}$ until the ion barely misses the carpet during its motion. This value was chosen as the maximum push field that the carpet can sustain. Since this is different, but related to the value of $E_{p, 50 \%}$ that was measured, a multiplicative factor was applied to the maximum push field to compare the two values. As indicated in Fig. 5, Eq. 1 correctly predicts the behavior of the measured push field for RF amplitude greater than 30 V. For lower amplitudes however, Eq. 1 significantly overestimates the maximum push field. Comparing the ion motion at $V_{R F}=10 \mathrm{~V}$ and $70 \mathrm{~V}$, shown in Fig. 6, gives an indication of what could be causing the discrepancy. Low RF amplitudes result in an ion motion that stays on average very close to the carpet while increasing the RF amplitude brings the ion further away from the carpet on average. Since the ion spends more time closer to the carpet at low RF amplitudes, it will have a greater probability of having a collision 

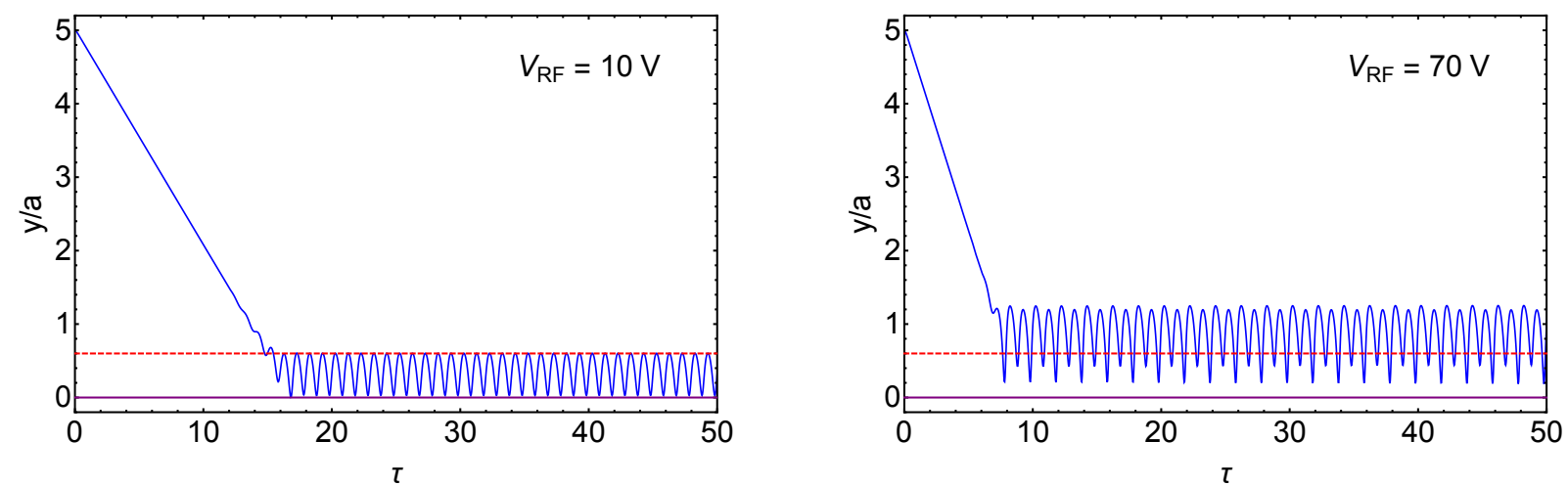

FIG. 6: Ratio of the distance of the ion from the RF carpet $y$ to the carpet pitch $a$ as a function of $\tau=f t$ for $V_{R F}=10 \mathrm{~V}$ (left) and $V_{R F}=70 \mathrm{~V}$ (right) in the cases where the ion barely misses the carpet. Note that for $V_{R F}=70 \mathrm{~V}$ the ion gradually gets closer to the carpet over a time period much longer then shown in the plot. The $y / a=0.6$ dashed line is given as a guide to the eye.

that will direct it to the carpet. Using this logic, the effect of these collisions (which are not included in Eq. 1) on the maximum push field can be mimicked by requiring the additional condition that the average distance of the ion above the carpet to be greater than a certain value. The dashed line in Fig. 5 shows the calculation results for the maximum push field when the ion's $y / a$ is both greater than zero at any given time as well as greater than 0.56 on average. The value $\langle y / a\rangle_{\max }=0.56$ was chosen as the one that better reproduced the behavior of the push field as function of RF amplitude and the push field as function of pressure.

Finally, Fig. 6 also gives an indication of why the behavior of $\varepsilon_{10 \mathrm{~V}}$ is highly correlated to $E_{p, 50 \%}$. As mentioned before, at small RF amplitudes the ion spends more time on average close to the carpet as compared to high amplitudes resulting in a greater probability of hitting the carpet, which is seen as a lower survival fraction.

\section{B. Push field vs. pressure of helium gas}

Measurements at different pressures were performed between 4 and 16 times at an average RF amplitude of $48 \mathrm{~V}$. Fig. 7 shows $E_{p, 50 \%}$ and $\varepsilon_{10 \mathrm{~V}}$ versus the pressure in the gas chamber. As shown, the tolerable push field increases with pressure up to about 100 mbar after which it 

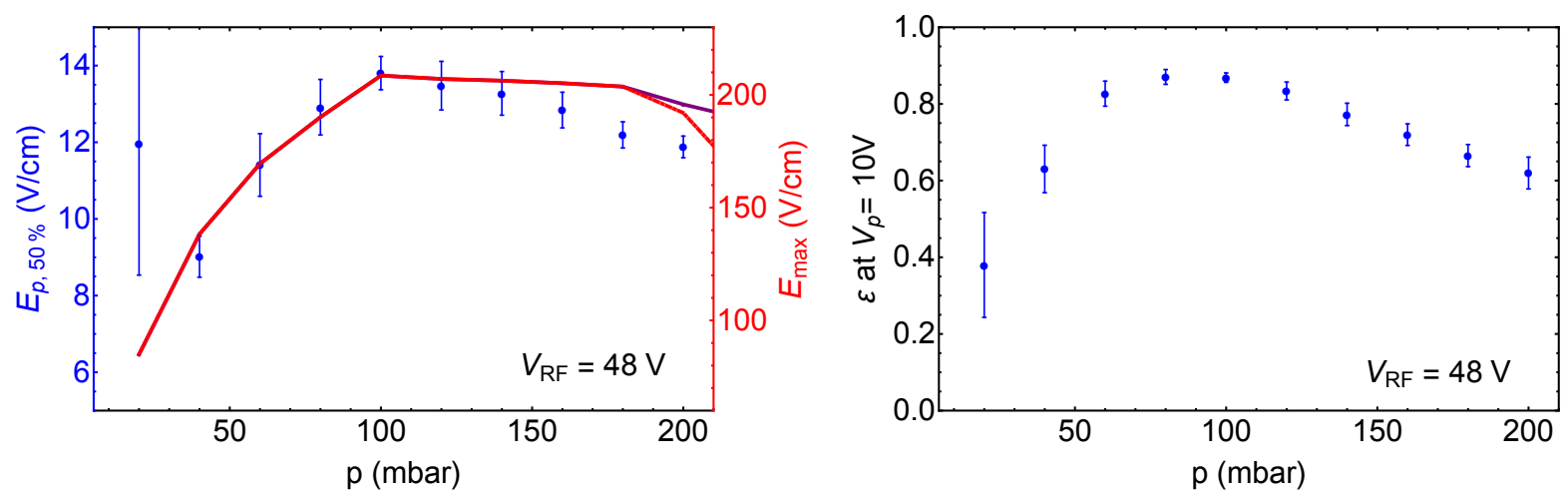

FIG. 7: Left: $E_{p, 50 \%}$ (circles) as a function of pressure. The purple curve is the maximum push field $E_{\max }$, when $y_{\pi}=0$, obtained by solving numerically eq. 1 . The red curve is similar to the solid curve but with the additional requirement that the average distance of the ion above the carpet must be above $0.57 \mathrm{~mm}$. Right: $\varepsilon_{10 \mathrm{~V}}$ as a function of pressure.

starts to slowly decline. This behavior was investigated by solving Eq. 1 numerically as done in the previous section. The solid curve in Fig. 7 gives the scaled results for the maximum push field obtained when the ion barely misses the carpet. The dashed curve is the same but with the added requirement that the ion must be on average above $y / a=0.56$. Note that both curves match the data fairly well except for a small over prediction of the maximum push field at pressures above 160 mbar. The decrease in efficiency at higher pressures might be due to more collisions that randomly direct the ions onto the carpet electrodes due to the increased collision rate at higher pressure. However, the mean free path also decreases at higher pressures, which may counteract that effect. A more thorough analysis of this effect would be needed. Hence the value $\langle y / a\rangle_{\max }=0.56$ should not stay constant with pressure as assumed in Fig. 7. The increased collisions at higher pressure would then result in an increase of $\langle y / a\rangle_{\max }$ and a lowering of the tolerable push field. Fig. 8 shows the position of the ion above the RF carpet over time. As indicated in the left figure, the decrease in tolerable push field with pressure under 100 mbar is caused by an increasingly unstable ion motion. The large "unpredictable" oscillations are a result of the ion motions being insufficiently damped at low pressures. Even if the ion is on average far from the carpet, the large oscillations of its motion are sufficient to bring the ion to an electrode. On the other hand at higher pressure (see Fig. 8 right), damping becomes sufficient to allow a stable and 

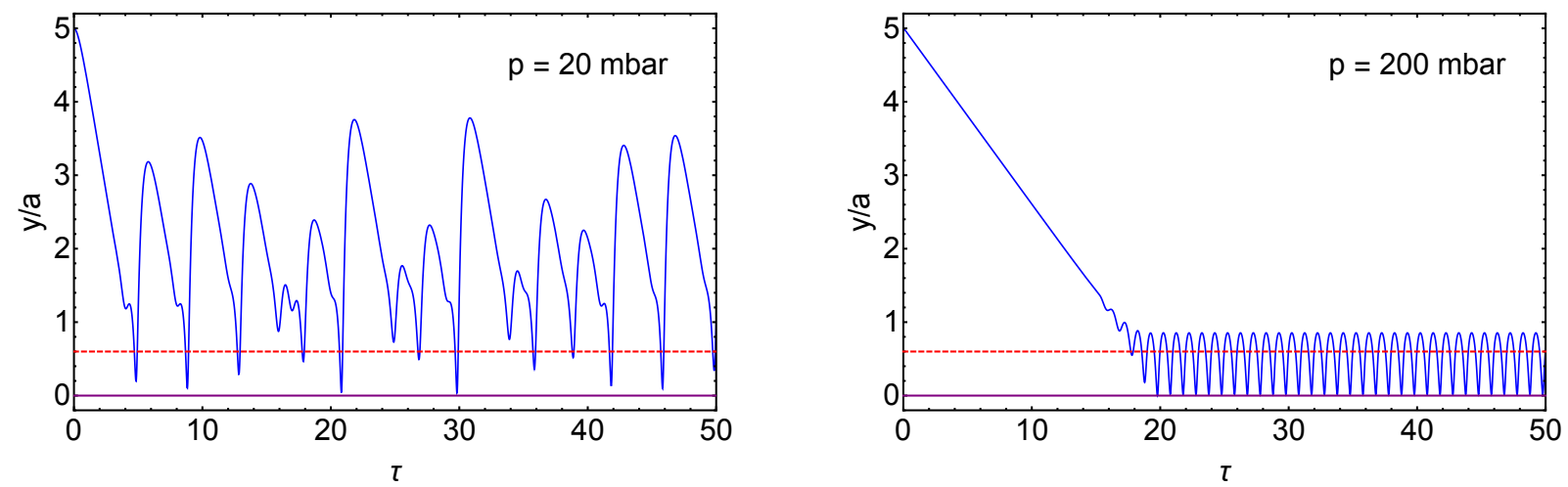

FIG. 8: Ratio of the distance of the ion from the RF carpet $y$ to the carpet pitch $a$ as a function of $\tau=f t$ for $p=20 \mathrm{mbar}$ (left) and $p=200 \mathrm{mbar}$ (right) in the cases where the ion barely misses the carpet. Note the highly unstable ion motion when $p=20$ mbar. The $y / a=0.6$ dashed line is given as a guide to the eye.

predictable ion motion. It should be noted that greater carpet pitches have been observed to offer a better performance at lower pressure [18].

Finally, Fig. 7 shows that $E_{p, 50 \%}$ and $\varepsilon_{10 \mathrm{~V}}$ follow a similar general trend with pressure. One feature, however, does stand out; $\varepsilon_{10 \mathrm{~V}}$ decreases more sharply than $E_{p, 50 \%}$ with pressure higher than $p=100$ mbar. This effect, also noticeable at low RF amplitude (see Fig. 5), might be due to the fact that by being much closer to the carpet on average at low $V_{R F}$ or high $p$, leads to a greater fraction of the ions being lost on the carpet even at a low $V_{p}$.

\section{CONCLUSION}

The behavior of the maximum repelling force was investigated and compared to theoretical predictions [13] as a function of RF amplitude and gas pressure. Potassium ions produced by an ion source were directed to an RF carpet by an electric field $E_{p}$. The ions transported along the carpet towards an outer ring by diffusion were then recorded as a current. The fraction of the ions transported was observed to decrease with increasing $E_{p}$. The $E_{p}$ at $50 \%$ of the initial survival fraction was determined and observed to increase for increasing RF amplitude. This behavior agreed well with the theoretical prediction from [13] when the reduction in efficiency due to collisions is mimicked by requiring the average 
ion position-to-carpet pitch to be above 0.56 in the calculation. A good general agreement between experiment and theory was also observed for the behavior of the maximum push field as function of gas pressure between 20 and 200 mbar. The maximum push field was observed to rapidly increase with pressure up to 100 mbar after which it slowly decreased with pressure. The poor RF carpet performance at low pressure could be due to the more unstable and insufficiently damped ion motion. Future investigations of the RF carpet repelling force behavior with gas pressure for other ion mass, carpet pitch and RF frequency would be of interest.

\section{ACKNOWLEDGMENTS}

We would like to thank G. Savard and S. Schwarz for fruitful discussions and sharing their impedance matching transformer designs. This work was supported in part by the US National Science Foundation Grant No. PHY-1062819 and Grant No. PHY-1419765.

[1] Y. Blumenfeld, T. Nilsson and P. Van Duppen, Phys. Scr. T152, 014023 (2013).

[2] K. Blaum, J. Dilling, W. Nörtershäuser, Phys. Scr. T152, 014017 (2013).

[3] M. Wada et al., Nucl. Instr. and Meth. in Phys. Res. B 317, 450 (2013).

[4] M. Wada et al., Nucl. Instr. and Meth. in Phys. Res. B 204, 570 (2003).

[5] M. Ranjan et al., EPL 96, 52001 (2011).

[6] F. Arai et al., Int. J of Mass Spec. 362, 56 (2014).

[7] M. Brodeur, A.E. Gehring, G. Bollen, S. Schwarz, D.J. Morrissey, Int. J of Mass Spec. 336, 53 (2013).

[8] G. Savard et al., Nucl. Instr. and Meth. in Phys. Res. B 204, 582 (2003).

[9] C. Droese et al., Nucl. Instr. and Meth. in Phys. Res. B 338, 126 (2014).

[10] S Naimi et al., Int. J of Mass Spec. 337, 24 (2013).

[11] S.N. Anthony, D.L. Shinholt, and M.F. Jarrold, Int. J of Mass Spec. 371, 1 (2014).

[12] G. Bollen, Int. J of Mass Spec. 299, 131 (2011).

[13] S. Schwarz, Int. J of Mass Spec. 299, 71 (2011).

[14] http://www.cathode.com/ 
[15] http://www.compunetics.com/

[16] C.J. Adkins, An Introduction to Thermal Physics, Cambridge University Press (1987).

[17] H.W. Ellis et al., At. Data and Nucl. Data Tab., 17, 177 (1976).

[18] Y. Ito et al., JPS Conf. Proc. 6, 030112 (2015). 


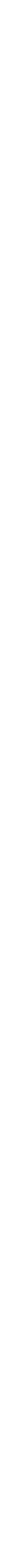

